\title{
Efficacy and In Vitro Cytotoxicity of Nanostructured Lipid Carriers for Paclitaxel Delivery
}

\author{
Shady Ali Swidan ${ }^{1,2^{*}}$, Hassan Mahmoud Ghonaim², Ahmed Mahmoud Samy ${ }^{3}$, Mamdouh Mostafa Ghorab ${ }^{2}$ \\ ${ }^{1}$ Department of Pharmaceutics, Faculty of Pharmacy, Sinai University, Al Arish, Egypt. \\ ${ }^{2}$ Department of Pharmaceutics, Faculty of Pharmacy, Suez Canal University, Ismailia, Egypt. \\ ${ }^{3}$ Department of Pharmaceutics, Faculty of Pharmacy, Al-Azhar University, Cairo, Egypt.
}

\begin{tabular}{l} 
ARTICLE INFO \\
\hline Article history: \\
Received on: $27 / 07 / 2016$ \\
Revised on: $13 / 08 / 2016$ \\
Accepted on: 01/09/2016 \\
Available online: $26 / 09 / 2016$ \\
\hline Key words: \\
Nanostructured lipid carriers, \\
Capryol 90, Paclitaxel, \\
Cytotoxicity, MCF7.
\end{tabular}

\begin{abstract}
Paclitaxel (PTX) is an anticancer drug having poor aqueous solubility and low bioavailability. Formulation of PTX into Nanostructure lipid carriers (NLC) could be a potential way to enhance PTX aqueous solubility and bioavailability hence increases efficacy and decreases side effects. Eight PTX-NLC formulae were prepared using homogenization-ultrasonication technique. Characterization of the nanoparticles was done by transmission electron microscopy and by measurement of particle size, poly dispersibility index and zeta potential. Encapsulation efficiency, drug loading, and In Vitro release were measured. Particle size ranged between 172.8 \pm 0.8 to $378.2 \pm 1.8 \mathrm{~nm}$ and zeta potential between $-18.6 \pm 0.4$ to $-28.1 \pm 1.2 \mathrm{mV}$. High EE and DL were obtained due to incorporation of liquid lipid and the In Vitro release showed prolonged time dependent release compared to Taxol ${ }^{\circledR}$. NLC-3 had the best results among the eight prepared formulae. In Vitro cytotoxicity of NLC-3 was evaluated on MCF-7 cell line and compared to pure PTX powder and Taxol ${ }^{\circledR}$. These findings show that NLC is a potential carrier to improve efficacy and enhance PTX delivery.
\end{abstract}

\section{INTRODUCTION}

In the recent years, there are many studies showed that the use of nanotechnology in the development of novel drug delivery systems could enhance the efficacy and alleviate the severe side effects accompanied with chemotherapy (Liu et al., 2011; Lønning, 2003). Paclitaxel (PTX) is an antitubulin chemotherapeutic agent derived from the bark of Taxus brevifolia the Pacific yew tree (Xin et al., 2010). The chemical structure of PTX does not contain enough ionizable or polar groups which limit its aqueous solubility $(<0.3 \mu \mathrm{g} / \mathrm{ml})$ (fig. 1) (Narvekar et al., 2014).In order to increase its water solubility, the commercially available PTX formulations use a mixture of Cremophor $\mathrm{EL}^{\circledR}$ and $50 \%$ dehydrated absolute ethanol in which PTX is solubilized (Cavalli et al., 2000). In addition to the wellknown side effects accompanied with PTX as a chemotherapeutic agent, the use of Cremophor $\mathrm{El}^{\circledR}$ causes severe hypersensitivity reactions and may cause shock syndrome in

Corresponding Author

Email: shady.swidan@su.edu.eg human after intravenous injection in addition to neurotoxicity, and nephrotoxicity (Hureaux et al., 2009; Zeng et al., 2012). Therefore, it is desired to formulate PTX into a novel delivery system to avoid the previously mentioned side effects. Nanotechnology and nano delivery systems are considered efficient means to achieve this goal. Different techniques were used to develop nanocarriers for delivery of chemotherapeutic agents, these include quantum dots, dendrimers and polymeric carriers (Hoskins et al., 2010; Lala and Awari 2014; Priya and Iyer 2015).

The use of lipid-based nanocarriers is considered the most successful technique for anticancer drug delivery due to high lipid miscibility of hydrophobic drugs in the lipid carriers such as liposomes, nanoemulsions, and solid lipid nanoparticles (SLN) (Hoskins et al., 2010; Priya and Iyer 2015; Swidan et al., 2016). Nanostructured lipid carriers (NLC) are the improved second generation of SLN. It was developed to overcome the drawbacks of SLN some of them are the decreased encapsulation efficiency (EE) and drug loading (DL) caused by drug leakage upon storage due to crystallization of the highly purified solid lipids forming the matrix of the nanocarrier into perfect crystals (Das and Chaudhury 2011). 
This offers limited space for the drug to be incorporated. The aforementioned problems can be overcome in NLC by the addition of spatially incompatible liquid lipid (oil) which distort the crystalline structure leading to the formation of imperfect crystals. This offers more spaces for the accommodation of the drug, thus NLC can enhance both EE and DL upon storage (Miao et al., 2015). In addition to the previous advantages, NLC is biocompatible, biodegradable, and applicable to large scale production. It can also prolong the drug release and circulation time of anticancer drugs thus accumulate them into the cancer tissue by enhanced permeability and retention effect (EPR) (Yang et al., 2013). All these advantages make NLC an optimum parenteral delivery system for PTX.

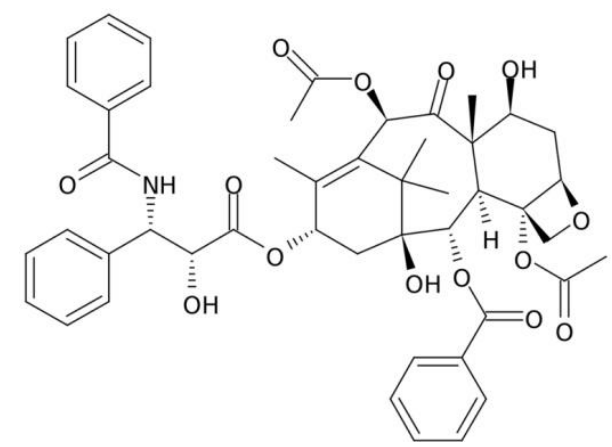

Fig. 1: Chemical structure of PTX.

In this study, PTX loaded NLC (PTX-NLC) were developed using the biocompatible solid lipid Glycerylmonostearate (GMS), while two different liquid lipids were used (Oleic acid \& Capryol 90). Two different SAA were incorporated (Tween $80 \&$ Poloxamer 188) each of higher or lower concentrations. The PTX In Vitro release and In Vitro cytotoxicity on human adenocarcinoma breast cancer cell line were evaluated to ensure both efficacy and safety of PTX loaded in this novel nanocarriers.

\section{MATERIALS AND METHODS}

\section{Materials}

PTX was purchased from MedKoo Biosciences Inc. (Chapel Hill, NC, USA). GMS, Poloxamer 188 (Pluronic F68), Tween 80 and oleic acid were provided by Sigma Aldrich (St. Louis, MO, USA). Capryol 90 (Propylene glycol monocaprylate) was a kind gift of Gattefosse (Saint-Priest, Cedex, France); Acetonitrile HPLC grade was purchased from Lab-Scan (Gliwice, Poland). Methylthiazolyldiphenyltetrazolium (MTT) and other chemicals for cell culture experiments were purchased from Sigma Chemical Company (St. Louis, MO, USA). All listed chemicals were used as supplied without modifications.

\section{Methods}

\section{Preliminary Fourier Transform Infrared Spectroscopy}

Fourier Transform Infrared Spectroscopy (FTIR) was done prior to formulation to ensure physical and chemical compatibility between the drug and the solid lipid used in the preparation of NLC formulae. In this analysis, Pure PTX, Pure GMS, and a 1:1 physical mixture of them were previously ground and mixed thoroughly with spectral grade potassium bromide. The $\mathrm{KBr}$ discs were prepared by compressing the powders. The scanning range was from 4000-400 $\mathrm{cm}^{-1}$. FTIR spectra were obtained using a Shimadzu 435 U-O4 IR spectrometer and the spectra were studied to ensure the presence of characteristic peaks.

\section{Production of PTX-NLC}

PTX-NLC formulae were prepared by emulsificationultrasonication method as described in (Nair et al., 2010; Riehemann et al., 2009)with some modifications. In brief, PTX was dissolved in the oily phase composed of GMS and one of the liquid lipids Capryol 90 or oleic acid in an amber glass vial. The oily phase in the vial was heated to $80^{\circ} \mathrm{C}$, a temperature which exceeds the melting point of the GMS. The surfactant (Tween 80 or Poloxamer 188) was dissolved in defined amount of water in a small beaker and the aqueous phase was then heated to the same temperature of the oily phase.

The aqueous phase containing the hydrophilic surfactant was dispersed in the oily component and homogenized using Heidolph silent crusher ${ }^{\circledR}$ homogenizer (Germany) for $10 \mathrm{~min}$ at $20000 \mathrm{rpm}$. This resulted in a coarse emulsion which is then converted to PTX-NLC with the desired size by sonication with Branson sonifier $^{\circledR} 450$ (CT, USA) for 15 minutes at sonication power of $90 \%$ of the maximum output. After sonication, nanoparticles were cooled for 2 hours at $4 \pm 0.5^{\circ} \mathrm{C}$. The composition of the eight nanoparticles formulae produced is listed in table 1.

Table 1: Composition of the eight prepared PTX-NLC.

\begin{tabular}{lcccccc} 
& $\begin{array}{c}\text { PTX } \\
(\boldsymbol{m} \boldsymbol{M})\end{array}$ & $\begin{array}{c}\text { GMS } \\
(\mathbf{m g})\end{array}$ & $\begin{array}{c}\text { Capryol } \\
\mathbf{9 0} \\
(\mathbf{m g})\end{array}$ & $\begin{array}{c}\text { Oleic } \\
\text { acid } \\
(\mathbf{m g})\end{array}$ & $\begin{array}{c}\text { Tween } \\
\mathbf{8 0} \\
(\mathbf{m g})\end{array}$ & $\begin{array}{c}\text { Poloxamer } \\
\mathbf{1 8 8} \\
(\mathbf{m g})\end{array}$ \\
\hline NLC-1 & 0.58 & 70 & 30 & - & 50 & - \\
NLC-2 & 0.58 & 70 & 30 & - & 100 & - \\
NLC-3 & 0.58 & 70 & 30 & - & - & 50 \\
NLC-4 & 0.58 & 70 & 30 & - & - & 100 \\
NLC-5 & 0.58 & 70 & - & 30 & 50 & - \\
NLC-6 & 0.58 & 70 & - & 30 & 100 & - \\
NLC-7 & 0.58 & 70 & - & 30 & - & 50 \\
NLC-8 & 0.58 & 70 & - & 30 & - & 100 \\
\hline
\end{tabular}

\section{Characterization of Lipid Nanoparticles \\ Transmission electron microscopy examination}

The morphology of the PTX-NLC particles was evaluated by Transmission Electron Microscopy (TEM) (model JTEM-1010, JEOL ${ }^{\circledR}$, Tokyo, Japan). The measurements were done by negative-staining method (Huang et al., 2010). In brief, one drop of the dispersion of the nanoparticle was put on copper grid coating, and then the excess droplets were removed using filter paper. After 5 minutes, one drop of uranylacetate solution $(2 \%$ w/v) was then dropped onto the grids. After the samples being negatively stained and air-dried at room temperature, they were ready for the TEM investigation which was done at $74 \mathrm{kV}$. 


\section{Particle Size Analysis}

Particle size analysis is a method to determine the particles' mean diameter and the index of polydispersity (PI) which is a measure of the uniformity of size distribution (Das and Chaudhury 2011). Particle size analysis of PTX-NLC was performed by dynamic light scattering (DLS) using a photon correlation spectrometer (Zetasizer, Malvern Instruments LTD, $\mathrm{UK})$ at $25^{\circ} \mathrm{C} \pm 1$, with detection limit $0.1-2000 \mathrm{~nm}$. All settings and analysis measurements were controlled using standard operation procedures. First, the samples of nanoparticle dispersions were diluted with equal amount of deionized water, the diluted dispersions were then added to the sample dispersion unit and all measurements were done using a beam length of $2.4 \mathrm{~mm}$ and a 45-mm focus objective.

\section{Measurement of the surface charge of PTX-NLC}

The electric charge on the surface of the nanoparticles is an indication of the physical stability of the colloidal system (Dubey et al., 2012). The surface charge of the prepared PTXNLC was measured using zeta potential by determining the electrophoretic mobility using the Malvern Zetasizer 3000 HSA (Malvern Instruments, UK). Samples were adjusted to a conductivity of $50 \mathrm{IS} / \mathrm{cm}$ with sodium chloride solution $(0.9 \%$ $\mathrm{w} / \mathrm{v}$ ) and measured in double distilled water. The $\mathrm{pH}$ was in the range of $5.5-7.5$ and the applied field strength was $20 \mathrm{~V} / \mathrm{cm}$.

\section{HPLC Analysis of PTX}

The HPLC system used for quantitative determination of PTX composed of Hitachi LaChrom Elite ${ }^{\circledR}$ HPLC system (Tokyo, Japan). The instrument was equipped with a model series L-2300 column oven and L-2000 organizer box. The pump is L-2130 pump with built in degasser andRheodyne $7725 \mathrm{i}$ injector with a 201 loop. The detector was L-2455 photo diode array detector (DAD) and the quantitation was made on a 250 x $4.6 \mathrm{~mm}$ (i.d.), $5 \mu \mathrm{m}$ ODS column (Inertsil, Tokyo, Japan). The HPLC was operated by EZchrom Elite version 3.3.2 SP1 by agilent. The mobile phase, acetonitrile-water $(57: 43$, v/v), flow rate 1.0 $\mathrm{ml} / \mathrm{min}$. Sample absorption was measured at wave-length $227 \mathrm{~nm}$. The sample injection volume was $60 \mu \mathrm{l}$. The column temperature was maintained at $25^{\circ} \mathrm{C} \pm 0.5$. Prior to use, the mobile phase was filtered through a $0.45 \mu \mathrm{m}$ hydrophilic membrane filter.

\section{Determination of PTX EE \& DL Capacity}

Both EE and DL was assessed indirectly, first the amount of free PTX (non-encapsulated) was removed and determined separately. EE and DL were determined as described in (Koning and Krijger 2007) with some modifications. The desired amounts of PTX-NLC were dispersed in phosphate buffer solution (PBS) with pH 7.4 containing $2 \%$ (wt) Tween 80 . They were then vortexed for 5 minutes to dissolve the free drug. The dispersion was centrifuged for $30 \mathrm{~min}$ at $15000 \mathrm{rpm}$ (Centrifuge model 216P, Sigma, Germany). The centrifugation led to the separation of the free PTX from PTX-NLC nanoparticles. After separation the samples were suitably diluted, the amount of the free PTX in the dispersion medium was estimated by HPLC as previously described. PTX EE and DL were calculated from the following equations:

$$
\begin{aligned}
& \operatorname{EE}(\%)=\frac{W_{\text {total }(\text { PTX })}-W_{\text {free }(P T X)}}{W_{\text {total }(\text { PTX })}} X 100 \\
& \text { DL }(\%)=\frac{W_{\text {total }(\text { PTX })}-W_{\text {free }(\text { PTX })}}{W_{\text {total (Lipids }+ \text { PTX })}} X 100
\end{aligned}
$$

$\mathbf{W}_{\text {total(PTX) }}, \mathbf{W} \mathbf{f}_{\text {ree(PTX) }}$ and $\mathbf{W}_{\text {total(lipids+PTX) }}$ are the weight of PTX added in the system, analyzed weight of PTX in supernatant and weight of the total lipids plus total PTX added in the system, respectively (Orive et al., 2009).All procedures are repeated three times and the mean \pm SD of EE and DL were calculated.

The addition of $2 \%$ by weight Tween 80 to the buffer was to dissolve the free PTX in the nanoparticle suspensions. The solubility of PTX in $2 \%$ Tween 80 -phosphate buffer solution was 28 micrograms per $\mathrm{ml}$ (Koning and Krijger 2007). The dilution of the nanoparticle dispersions with distilled water was to ensure that the total amount dissolved in the solution is less than $28 \mu \mathrm{g} / \mathrm{ml}$.

\section{In Vitro Release of PTX}

The In Vitro drug release from PTX-NLC was evaluated using the dialysis bag method (Kuo and Chung 2011) with slight modifications. The dialysis membrane was soaked for 24 hours in phosphate buffer, pH 7.4 containing 2\% Tween-80 (the release medium) before the experiment. Accurately measured one milliliter of the PTX-NLC formulations or one ml of Taxol ${ }^{\circledR}$ was placed in the dialysis bag. The membrane was thoroughly tied to prevent leakage of the drug. The dialysis bag was put in a beaker containing $30 \mathrm{ml}$ of the release medium. The beaker was kept horizontally on a Clifton ${ }^{\circledR}$ shaking water bath (Nickel-Electro LTD, United Kingdom) rotating at $100 \mathrm{rpm}$ for 48 hours. The beaker was protected from light and $5 \mathrm{ml}$ release medium samples were withdrawn at predetermined time intervals, and replaced by the same volume of fresh release medium to maintain sink conditions.

Samples were diluted to a suitable concentration, then analyzed using HPLC. The experiment was done in triplicates and the mean \pm SD was determined.

\section{Differential Scanning Calorimetry Analysis}

Differential Scanning Calorimetry (DSC) analysis was done to study the physical state and polymorphism of the PTXNLC. The measurements were performed using differential scanning calorimeter, Shimadzu DSC-50, (Japan). Samples weighing 2-5 mg were heated and scanned between $25^{\circ} \mathrm{C}$ and $250^{\circ} \mathrm{C}$ and a heating rate of $10^{\circ} \mathrm{C} \cdot \mathrm{min}^{-1}$ under nitrogen gas flow (30 ml. $\mathrm{min}^{-1}$ ) (Peer and Margalit 2006). DSC analyses were performed on the bulk solid lipid GMS, the pure drug (PTX), physical mixture (1:1) and all prepared PTX-NLC formulae. 


\section{In Vitro Cytotoxic Assay}

From all previous tests and experiments, all PTX formulae were ranked and the best formula is selected for In Vitro cytotoxicity study. The cytotoxicities of the best selected PTXNLC formula, pure PTX, and Taxol ${ }^{\circledR}$ were investigated against human adenocarcinoma breast cancer cell line MCF-7. The cytotoxic effect was evaluated using the MTT (3-[4, 5dimethylthiazole-2-yl]-2, 5-diphenyltetrazolium bromide) dye reduction assay (Fang et al., 2015). Briefly, in serum-free media, cells $(0.5 \times 105$ cells/ well) were plated in a flat bottom 96 wells microplate, and treated with $20 \mu \mathrm{l}$ of different concentrations of the tested samples for $48 \mathrm{~h}$ at $37 \pm 1^{\circ} \mathrm{C}$, in a humidified $5 \% \mathrm{CO}_{2}$ atmosphere. After incubation, media were removed and $40 \mu \mathrm{l}$ MTT solution per well were added and incubated for an additional $4 \mathrm{~h}$. MTT crystals were solubilized by adding $180 \mu \mathrm{l}$ of acidified isopropanol/well and the plate was stirred at room temperature, followed by photometric determination of the absorbance at 570 $\mathrm{nm}$ using microplate enzyme linked immunosorbent assay (ELISA) reader. Triplicate repeats were performed for each concentration and the average was calculated. Data were expressed as the fraction of survival compared with the untreated cells compared with the vehicle control, with cytotoxicity indicated by fraction relative viability. The fraction of survival was calculated according to the following equation:

\section{Absorbance of treated cells}

Absorbance of control cells

Then the half maximal inhibitory concentrations (IC50) for all samples were calculated from the equation of the dose response curve.

\section{RESULTS AND DISCUSSIONS}

\section{FTIR analysis}

The FTIR spectroscopy is done on pure PTX powder and GMS and the binary physical mixture of the drug and the solid lipid to investigate possible incompatibilities may occur before formulation.

As shown in (Fig. 2) the FTIR spectra of PTX, GMS and a binary mixture of equal ratios of both ingredients respectively. PTX spectrum shows different characteristic vibrational peaks of PTX. Peaks number 4 and 5 at 3485 and $3439 \mathrm{~cm}^{-1}$ respectively are specific for $\mathrm{N}-\mathrm{H}$ and $\mathrm{O}-\mathrm{H}$ stretching. Peaks in the range of 2900 and $3100 \mathrm{~cm}^{-1}$ are characteristic peaks for $\mathrm{CH}_{3} / \mathrm{C}-\mathrm{H}$ stretching. It also had characteristic peak number 17 at $1646 \mathrm{~cm}^{-1}$ which is corresponding to carbonyl group $(\mathrm{C}=\mathrm{O})$. According to the chemical structure of GMS, it has characteristic hydroxyl functional group which shows $\mathrm{O}-\mathrm{H}$ stretching vibration at 3306 $\mathrm{cm}^{-1}$ as a broad peak (peak number 11). In the binary mixture spectrum, a combination of characteristic peaks for the functional groups in both substances is observed. The peaks corresponding to the $\mathrm{N}-\mathrm{H} / \mathrm{O}-\mathrm{H}$ stretching of PTX and the $\mathrm{O}-\mathrm{H}$ stretching vibration of GMS are diffused in a wider peak at $3311 \mathrm{~cm}^{-1}$. Peaks corresponding to the carbonyl group, $\mathrm{CH}_{3}$ and $\mathrm{C}-\mathrm{H}$ stretching appeared in the same wave number range as the pure PTX powders. This indicates that both drug and lipid remained unchanged.

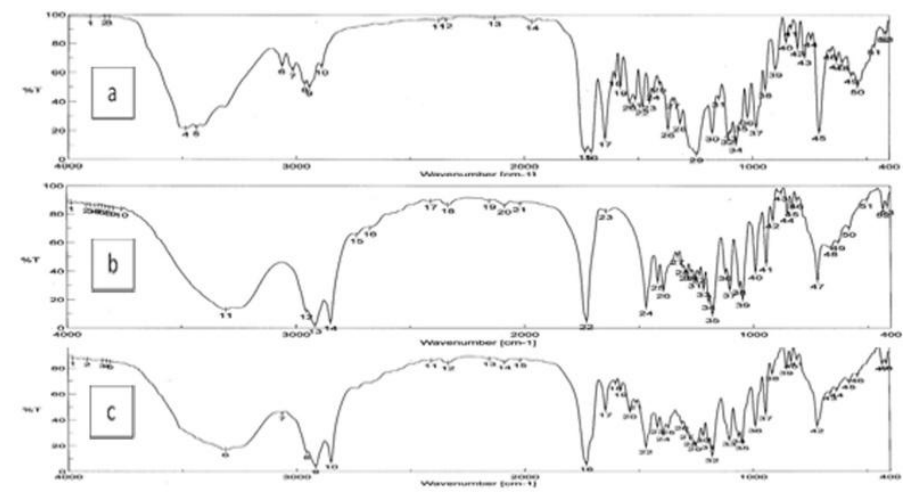

Fig. 2: FTIR spectra of a) PTX b) GMS c) binary physical mixture of PTX \& GMS (1:1)

\section{Preparation of PTX-NLC}

All PTX-NLC were prepared successfully using the homogenization ultra-sonication technique. Capryol 90 and oleic acid are used as liquid lipids, although Capryol 90 is rarely used as a liquid lipid in NLC - as it is commonly used in self-emulsifying preparations - it was used efficiently in NLC preparations. There was neither physical separation nor chemical incompatibility between Capryol 90 and all other constituents used in the formulation.

\section{Characterization of the prepared PTX-NLC nanoparticles Transmission Electron Microscopy}

The TEM photographs of the prepared PTX-NLC formulae are shown in (Fig. 3) in which lipid nanoparticles were magnified by 80000 times. From these figures, it is obvious that nanoparticles in all formulations are mainly discrete entities. All particles, irrespective of the formulation investigated were in the nanometer range.
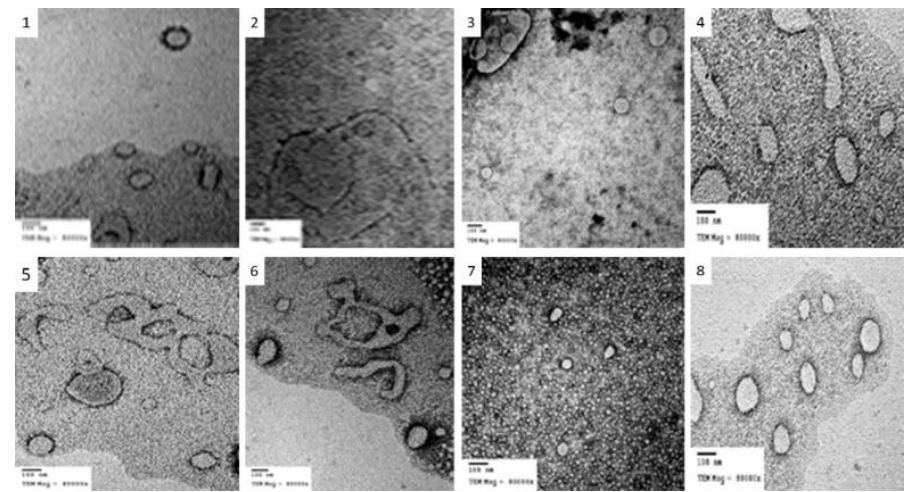

$\prod_{i=1}$

Fig. 3: TEM photographs of the eight prepared NLC formulae (NLC1-NLC8).

The TEM investigation also revealed that the PTX-NLC nanoparticles were homogeneous and spherical or ellipsoidal in shape. This indicates good homogeneity and good uniformity that 
may result in the uniform release of the entrapped PTX. Slight difference in particle size was observed when particle diameter was measured using TEM technique and photon correlation technique. Particles had a smaller particle size in TEM measurement as the process involving drying of the sample, while in PC hydration of samples maintained the size of the nanoparticles (Liu et al., 2007; Sanad et al., 2010).

\section{Particle size and poly dispersibility index}

Particle diameter in the prepared PTX-NLCs ranged from $172.8 \pm 0.8 \mathrm{~nm}$ to $378.2 \pm 0.8 \mathrm{~nm}$, which were the values of particles diameter in formulae NLC-7 and NLC-5 respectively. Values of Polydispersibility index ranged from $0.251 \pm 0.01$ and $0.652 \pm 0.07$ for formulae NLC-5 and NLC-7 respectively. From the data obtained, it is obvious that the use of Capryol 90 compared to oleic acid caused a decrease in the particle size which could be related to the higher viscosity of oleic acid at $20^{\circ} \mathrm{C}(36.56$ cps) when compared Capryol 90 (30 cps) at the same temperature (Viswanath et al., 2007). According to literature, the better polydispersibility index range is $<0.5$ which means that five of the eight formulae had excellent particle size distribution, while the other three had a relatively wide range of particle size. More optimization of sonication time could be necessary to narrow the range of particle size distribution in these formulae.

\section{Zeta Potential of PTX-NLC}

The surface charge generates a potential around the particle, this potential is at the highest at the surface and decreases with distance into the medium. This potential is called zeta potential, which plays an important role in the stability of dispersions owing to the repulsion of particles which prevents the aggregation into larger particles. Zeta potential of all prepared PTX-NLCs was negative due to the use of negatively charged GMS as solid lipid and the use of oleic acid or Capryol 90 as liquid lipid.

As seen in Table 2, zeta potential values of the eight prepared NLCs ranged between $-28.4 \mathrm{mV}$ and $-18.6 \mathrm{mV}$ for formulae NLC-5 and NLC-2 respectively. It is clear that high zeta potential - close to $-30 \mathrm{mV}$ - may enhance stability of the nanoparticles in addition to the steric stabilization of nanoparticles due to the use of Poloxamer 188 and Tween 80 as surfactants. These surfactants stabilize NLCs by forming a coat around their surface and decrease the electrostatic repulsion between the particles. This effect may enhance the role of repulsive effect of zeta potential; thus enhancing the physical stability of the nanoparticles and prevent their aggregation. Some researchers have found that zeta potential of the NLC was $-25 \mathrm{mV}$, and it is sufficiently high to form stable colloidal nanosuspension (Velmurugan and Selvamuthukumar 2015).

The type of liquid lipid has an obvious effect on zeta potential. The use of oleic acid resulted in an increase in the negative charge on the surface of molecules higher than that when Capryol 90 is used. In the four pairs of comparison Capryol 90 contained in NLCs had lower zeta potential value. This may be due to the presence of a strong carboxyl group with strong negative charge in oleic acid which is not present in the structure of Capryol 90, which increases the charge resulted from the GMS so NLCs contain oleic acid had higher negative charge.

Table 2: Particle size (PS), polydispersibility index (PDI) and zeta potential of the prepared PTX-NLC.

\begin{tabular}{cccc}
\hline & PS $(\mathbf{n m})$ & PDI & ZP(mV) \\
\hline NLC-1 & $248.1 \pm 3.1$ & $0.61 \pm 0.01$ & $-24.9 \pm 0.5$ \\
NLC-2 & $219.6 \pm 0.9$ & $0.64 \pm 0.03$ & $-18.6 \pm 0.4$ \\
NLC-3 & $210.2 \pm 2.7$ & $0.45 \pm 0.04$ & $-21.0 \pm 0.6$ \\
NLC-4 & $288.5 \pm 2.8$ & $0.43 \pm 0.03$ & $-22.0 \pm 0.1$ \\
NLC-5 & $378.2 \pm 1.8$ & $0.25 \pm 0.01$ & $-28.1 \pm 1.2$ \\
NLC-6 & $276.0 \pm 2.3$ & $0.33 \pm 0.02$ & $-26.0 \pm 0.8$ \\
NLC-7 & $172.8 \pm 0.8$ & $0.65 \pm 0.07$ & $-22.1 \pm 1.0$ \\
NLC-8 & $317.1 \pm 1.1$ & $0.55 \pm 0.07$ & $-24.9 \pm 0.4$ \\
\hline
\end{tabular}

\section{HPLC Analysis of PTX}

PTX was found to have a good linear relationship in $2 \%$ w/v Tween 80 phosphate buffer solution $(\mathrm{pH} 7.4)$, when its concentration was between 1 and $20 \mu \mathrm{g} / \mathrm{mL}(\mathrm{r}=0.997)$. The HPLC technique was used to determine EE, DL and In Vitro release. A complete three-dimensional wave-length scan $(200-300 \mathrm{~nm})$ is shown in (Fig. 4). The $\lambda_{\max }$ was $227 \mathrm{~nm}$ and the retention time was $10.5 \mathrm{~min}$.

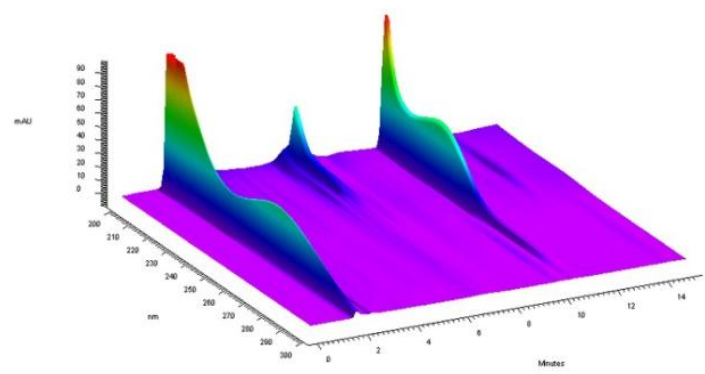

Fig. 4: Three dimensional wave length scan of PTX in PBS (pH 7.4) with $2 \%$ W/V tween 80 solution shows absorption and retention time.

\section{Determination of EE \& DL of PTX in NLC formulae}

From the data listed in table 3, the highest encapsulation efficiency value of PTX was found to be for NLC-3 with $89.1 \% \pm$ 1.5 , while the lowest value was in formula NLC- 8 by $77.3 \% \pm$ 3.3.

Table 3: Encapsulation efficiency and PTX loading capacity (DL) of PTXNLC

\begin{tabular}{ccc}
\hline & EE(\%) & DL(\%) \\
\hline NLC-1 & $78.9 \% \pm 2.5$ & $7.17 \% \pm 0.32$ \\
NLC-2 & $82.1 \% \pm 5.3$ & $7.46 \% \pm 0.29$ \\
NLC-3 & $89.1 \% \pm 1.5$ & $8.10 \% \pm 0.15$ \\
NLC-4 & $87.6 \% \pm 1.8$ & $7.96 \% \pm 0.09$ \\
NLC-5 & $81.3 \% \pm 2.3$ & $7.39 \% \pm 0.22$ \\
NLC-6 & $80.1 \% \pm 3.6$ & $7.28 \% \pm 0.32$ \\
NLC-7 & $78.9 \% \pm 2.7$ & $7.17 \% \pm 0.44$ \\
NLC-8 & $77.3 \% \pm 3.3$ & $7.03 \% \pm 0.41$ \\
\hline
\end{tabular}

The high EE of PTX in the NLCs could be attributed to the presence of the liquid nano-compartments formed by Capryol 
90 or oleic acid, which were entrapped within the solid matrix of the NLCs. So, NLCs served as nanoreservoir drug delivery systems for PTX. Similar results have been obtained by Liu et al., (2011) who have prepared NLC containing Docetaxel (DTX) which is structurally and therapeutically similar to PTX. They have found that the average EE of the freshly prepared DTX-NLC was $89.72 \pm 0.89 \%$, while Tiwari and Pathak have mentioned that the EE of nanoparticles was increased from $81.74 \%$ to $93.33 \%$ respectively upon increasing the percentage of oleic acid from 15 to $30 \mathrm{wt} \%$ (Tiwari and Pathak 2011).

Fig. 5 shows the effect of liquid lipid formulated in the NLCs formulation. It is clear that most formulae contain Capryol 90 had higher values of EE than those contain oleic acid as liquid lipid. This could be due to structural and spatial difference of Capryol 90 (Propylene glycol monocaprylate) and the solid lipid GMS leading to more imperfections in the matrix offering space to accommodate more PTX, while Oleic acid is a monounsaturated fatty acid form of stearic acid (Sharma et al., 2009).

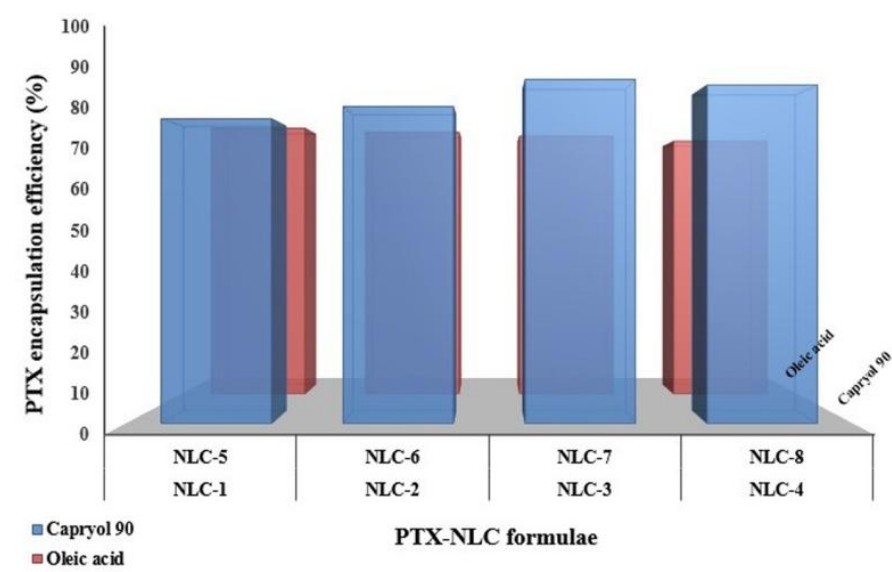

Fig. 5: Effect of the type of liquid lipid on the encapsulation efficiency of PTX in NLC.

The highest DL percentage was in NLC-3 with $8.1 \% \pm$ 0.15 , while the lowest value was in formula NLC- 8 by $7.03 \% \pm$ 0.41 . As the total weight of lipids was fixed in all formulae (100 $\mathrm{mg}$ ) with a fixed lipid ratio to the drug (10:1) respectively, no change in the rank order of PTX-NLCs formulae from that mentioned in EE section was observed.

These results are in agreement with the findings which have been obtained by different researchers. Liu and coworkers have shown that liquid lipids were added in lipids of NLC and the solubility of DTX in NLC dispersion increased up to $1.0 \mathrm{mg} / \mathrm{ml}$, this value is high enough to be used in clinical studies $(<0.74$ $\mathrm{mg} / \mathrm{ml}$ ). They have used oleic acid as liquid lipid for the preparation of the NLC (Liu et al., 2011).

Fig. 6 shows the effect of liquid lipid formulated in the NLCs formulation on DL. It is clear that most formulae that contain Capryol 90 had higher values of DL than those contain oleic acid as liquid lipid. The same cause mentioned in EE study could be responsible for the higher DL values in Capryol 90 formulae; which is the structural and spatial difference of Capryol 90 from GMS.

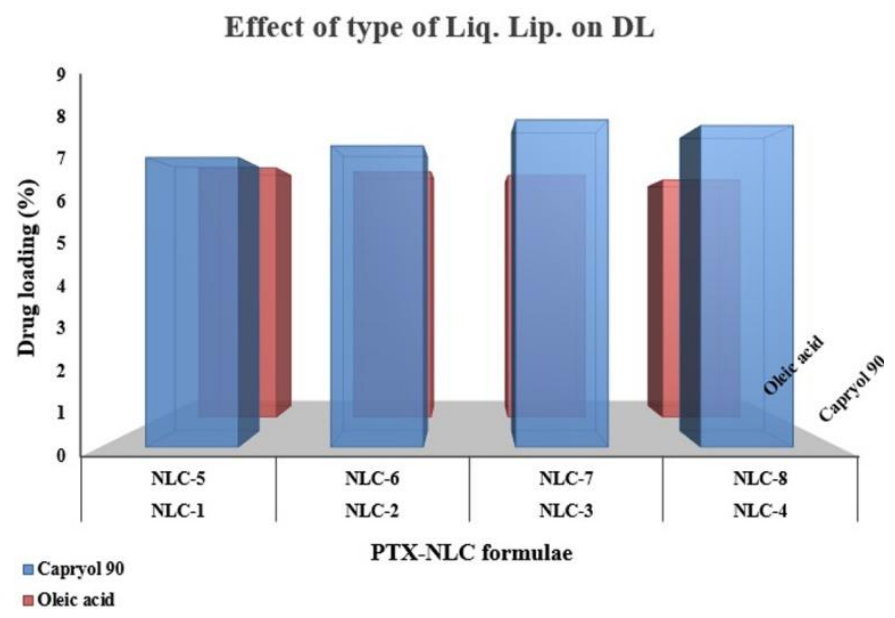

Fig. 6: Effect of the type of liquid lipid on the drug loading of PTX in NLC.

\section{In Vitro Release of PTX-NLC}

The In Vitro release profile was evaluated by dialysis bag for 48 hours at predetermined time intervals. The release profile of all NLCs formulae were described by plotting PTX percent release versus time compared to the PTX market product Taxol ${ }^{\circledR}$.

Fig. 7 shows the release profile of PTX-NLC formulae compared to Taxol $^{\circledR}$. Each four formulae were illustrated in a separate plot. As shown in the figure, the release profile of the drug from NLCs formulae was slower than that of Taxol ${ }^{\circledR}$. About 98.2\% of PTX was released from Taxol ${ }^{\circledR}$ after only 18 hours, while only $67.1 \%, 57.2 \%, 60.5 \%$ and $51.9 \%$ of PTX were released in the same period from formulae NLC-1, NLC-2, NLC-3 and NLC-4 respectively. At the end of sampling time ( $48 \mathrm{hrs})$ the release of PTX from the NLCs formulae was $78.1 \%, 72.1 \%, 65.1 \%$ and $63.5 \%$ from formulae NLC-1, NLC-2, NLC-3and NLC-4 respectively. It's clear that the release of PTX incorporated to NLC formulae was slower than that from Taxol ${ }^{\circledR}$.

Similar results were obtained in NLC-5 to NLC-8. After 18 hours - when almost all PTX released from Taxol ${ }^{\circledR}$ - the release of PTX from NLC-5 was $72.4 \%$, from NLC-6 was $66.4 \%$, and from NLC-7 was $55.5 \%$, while the release of PTX from NLC-8 was $65.6 \%$. After 48 hours the release of PTX from NLC-5, NLC6, NLC-7 and NLC-8 was $80.2 \%, 71.9 \%, 60.7 \%$ and $77.0 \%$ respectively. All formulae showed consistently, time-dependent and prolonged PTX release.

The difference between the release properties of PTX from Taxol ${ }^{\circledR}$ and from PTX-NLCs is evidently attributed to the prolonged release function of NLC. Lipophilic poorly water soluble PTX was held by the lipid core of the NLC and the drug released mainly through dissolution and diffusion. This result implied that PTX could be released slowly from PTX-NLC and 
could keep constant concentration for relatively longer period. Therefore, PTX-NLC may reduce the frequency of PTX administration. This is good for the clinical application and is a better step to overcome limitations accompanied by PTX administration.
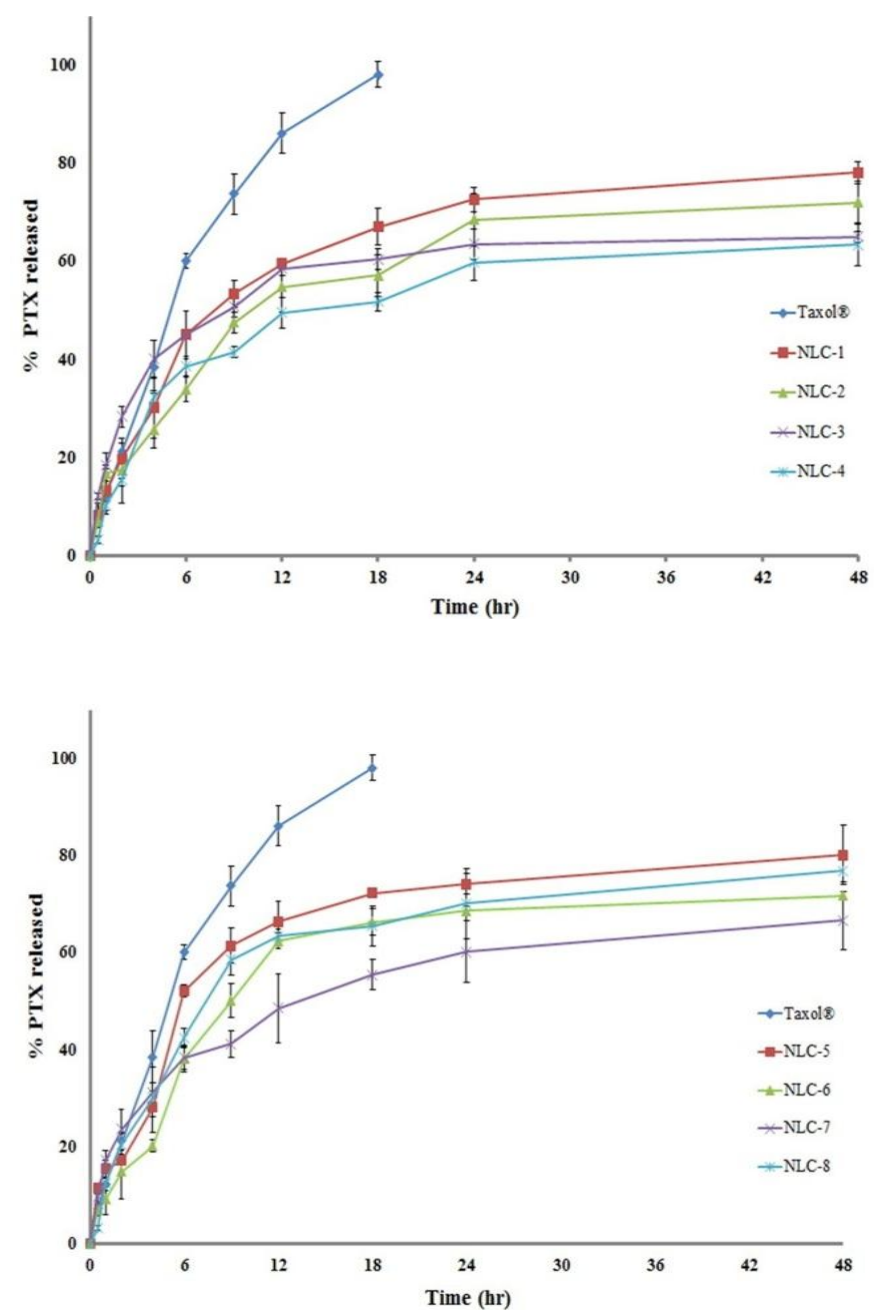

Fig. 7: In Vitro release of PTX form NLC compared to Taxol®. At top: NLC1 - NLC4. At bottom NLC5 - NLC8.

The release mechanism might be explained as follows: the liquid lipids were located at the outer shell of the nanoparticles besides distributed in solid-lipid core, so the liquid lipid-enriched outer layers could load a larger amount of drug which might be easily released. Hence, the nanoparticles exhibited rapid release at the initial stage and the drug incorporated into the solid-lipid core was released in a prolonged pattern of erosion or degradation of lipid matrix (Jia et al., 2012).

Similar findings have been obtained by Liu and colleagues who have found that formulation of DTX in NLCs shows a sustained release profile. It was obvious that DTX released much slower from DTX-NLC than from the commercially available DTX preparation Duopafei ${ }^{\circledR}$ (Liu et al., 2011).

\section{DSC study of PTX-NLC}

DSC study provides a detailed look at the crystallization behavior and melting of crystalline materials like lipid nanoparticles. Thermoanalytical studies were performed on PTXNLC formulae, compared to The thermogram of pure PTX and GMS. The main goal of this study was to detect if there any difference in the lipid matrix of each formula due to its composition.

The resultant sharp endothermic peak of PTX was at $220.06^{\circ} \mathrm{C}$. This is an intermediate value between the endothermic peak of PTX which has been determined by Liggins et al., (1997) and Zhang et al., (2010) which was $223{ }^{\circ} \mathrm{C}$, and the value determined by Jauhari et al., (2009) who have found that endothermic peak was at $217^{\circ} \mathrm{C}$. The enthalpy of PTX melting is $41.2 \mathrm{~J} / \mathrm{g}$ compared to $55.35 \mathrm{~J} / \mathrm{g}$ as have been stated in the same study.

The endothermic peak of GMS appeared at $69.1^{\circ} \mathrm{C}$, while the enthalpy of GMS is $173.25 \mathrm{~J} / \mathrm{g}$. These findings were similar to results obtained by Yahjma and coworkers who have mentioned that the endothermic peak of $\alpha$ form of GMS was at $67.9^{\circ} \mathrm{C}$, while the endothermic peak of the $\beta$ form appeared at $71.9^{\circ} \mathrm{C}$ (Yajima et al., 2002). Another close value was mentioned by Hussain and his colleagues who have stated that the endothermic peak of GMS was at $65.4^{\circ} \mathrm{C}$ (Hussain et al., 2013).

The physical mixtures showed the endothermic peak of the lipid and the drug with no shift indicating that both of them were in the crystalline form in the binary mixture in the ratio (1:1). The Thermogram of all prepared PTX-NLC formulae showed no endothermic peak at or around $220^{\circ} \mathrm{C}$ indicated that PTX was in (non-crystalline) or amorphous state in the NLCs formulae. This also indicated that there is complete miscibility of the drug with both solid and liquid lipids and good incorporation of molecular PTX into NLCs.

It was also noted that in NLC formulae: NLC-3, NLC-4, NLC-7 and NLC-8 there was an additional peak appeared around $50^{\circ} \mathrm{C}$ which is related to the solid surfactant Poloxamer 188. This peak did not appear in the liquid surfactant Tween 80 containing formulae.

In all PTX-NLC formulae, there was a sharp decline in enthalpy from the bulk material to the nanocarriers. For amorphous solids or less ordered crystals, the melting of the substance consumes much less energy than crystalline substances which need to overcome lattice forces (Hou et al., 2003).

From the obtained results, it has been concluded that the lipid in the nanoparticles should be in a less ordered arrangement compared to the bulk lipid. Furthermore, due to the incorporation of Capryol 90 or Oleic acid, NLC should be less ordered compared to SLN and the inner core of the PTX-NLC should be completely amorphous, which is consistent with the DSC analysis. In other words, the addition of the liquid lipid to the nanoparticles decreases the crystallinity of the lipid matrix. A less ordered crystal or amorphous lipid matrix would be favorable for encapsulating more drug molecules thus enhancing DL and 
prolonging the drug release of the lipophilic drug from the lipid matrix.

\section{The In Vitro Cytotoxicity assay of PTX-NLC}

From the previous experiments, the eight PTX-NLC formulae were ranked in ascending way according to mean particle size and descending according to zeta potential, EE and In Vitro release after 48 hours. NLC-3 had the highest rank and subjected to In Vitro cytotoxic assay to evaluate the therapeutic efficacy of the PTX nanoparticles compared to the market product and the pure PTX powder. The fraction of relative viability was calculated and IC50 was determined. Fractions remained of viable cells in each concentration $(0,5,21.5,25,50 \mu \mathrm{g} / \mathrm{ml})$ was shown in Fig. 8 . As seen from the figure, the IC50 of PTX powder was $21.4 \pm 0.27$ $\mu \mathrm{g} / \mathrm{ml}$, this high IC50 may be due to the very low solubility of PTX in water which caused marked decrease in PTX amount required for cell death. The In Vitro cytotoxicity assay of Taxol ${ }^{\circledR}$ solution with same concentrations used in pure PTX showed a significant decrease in the calculated IC50 with a value of $3.75 \pm$ $0.07 \mu \mathrm{g} / \mathrm{ml}(\mathrm{P}<0.05)$. The IC50 of NLC-3 was $3.53 \pm 0.11 \mu \mathrm{g} / \mathrm{ml}$, which is less than that of Taxol ${ }^{\circledR}$ and significantly lower than that of pure PTX powder $(\mathrm{P}<0.05)$.

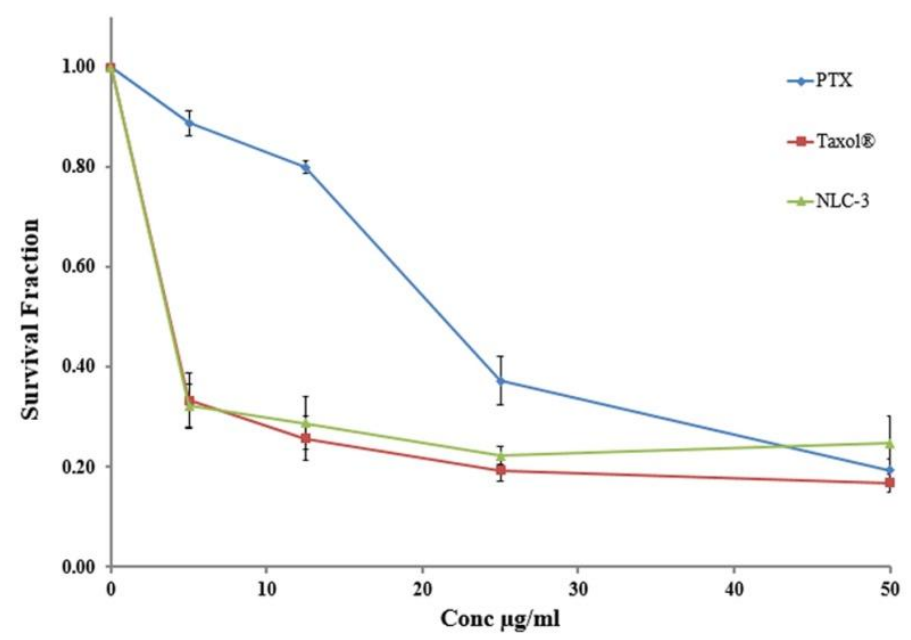

Fig. 8: In Vitro cytotoxicity of NLC3 compared to pure PTX and Taxol ${ }^{\circledR}$ in MCF-7 cells after $48 \mathrm{~h}$ exposure.

The incorporation of PTX into NLC resulted in a decrease in its particle size. Also, the lipid nature of the nanoparticles increases in the lipid matrix and the drug is in amorphous form. This may lead to an increase in PTX penetration into the tumor cell which increase its cytotoxic effect significantly. Thus PTX-NLC formula exhibited strong inhibitory effects on the proliferation of MCF-7 cells. Similar finding has been found in the literature with different nanoparticles, as according to the researchers' knowledge there is no published work evaluated PTX incorporated in NLC against MCF-7. Yao and colleagues have studied the cytotoxic effect of PTX formulated in nanomicelles on MCF-7 cell line, they have found that at various concentration points, functional PTX nanomicelles exhibited the strongest inhibitory effect on the proliferation of MCF-7 cells among various formulations (Yao et al., 2011).

\section{CONCLUSION}

PTX was successfully incorporated into NLC using the homogenization-ultrasonication technique. The addition of Capryol 90 as a liquid lipid resulted in a decrease in particle size and increase in EE and DL compared to Oleic acid containing formulae. PTX incorporated into NLC was in amorphous form as confirmed by DSC analysis. NLC-3 had the best overall rank compared to all prepared formulae and it had significantly lower IC50 compared to PTX and comparable to that of Taxol ${ }^{\circledR}$. Formulation of PTX in NLC enhanced its efficacy hence its parenteral dose can be lowered which increase its safety and minimized its side effects.

\section{Financial support and sponsorship: Nil.}

Conflict of Interests: There are no conflicts of interest.

\section{REFERENCES}

Cavalli R, Caputo O, Gasco MR. Preparation and characterization of solid lipid nanospheres containing paclitaxel. European journal of pharmaceutical sciences, 2000; 10:305-309.

Das S, Chaudhury A. Recent advances in lipid nanoparticle formulations with solid matrix for oral drug delivery. AAPS PharmSciTech, 2011; 12:62-76.

Dubey A, Prabhu P, Kamath JV. Nano Structured lipid carriers :A Novel Topical drug delivery system. International Journal of PharmTech Research, 2012; 4:705-714.

Fang R, Yang S, Wang Y, Qian H. Nanoscale Drug Delivery Systems: A Current Review on the Promising Paclitaxel Formulations for Future Cancer Therapy. NANO, 2015.

Hoskins C, Ouaissi M, Lima SC, Cheng WP, Loureirio I, Mas E, Lombardo D, Cordeiro-da-Silva A, Ouaissi A, Kong Thoo Lin P. In Vitro and in vivo anticancer activity of a novel nano-sized formulation based on self-assembling polymers against pancreatic cancer. Pharmaceutical research,2010; 27:2694-2703.

Hou D, Xie C, Huang K, Zhu C. The production and characteristics of solid lipid nanoparticles (SLNs). Biomaterials,2003; 24:1781-1785.

Huang Q, Yu H, Ru Q. Bioavailability and delivery of nutraceuticals using nanotechnology. Journal of food science, 2010; 75:50-57.

Hureaux J, Lagarce F, Gagnadoux F, Vecellio L, Clavreul A, Roger E, Kempf M, Racineux JL, Diot P, Benoit JP, Urban T. Lipid nanocapsules: ready-to-use nanovectors for the aerosol delivery of paclitaxel. European journal of pharmaceutics and biopharmaceutics,2009; 73(2): 239-246.

Hussain T, Saeed T, Mumtaz AM, Javaid Z, Abbas K, Awais A, Idrees HA. Effect of two hydrophobic polymers on the release of gliclazide from their matrix tablets. ACTA Poloniae Pharmaceutica-Drug Research, 2013; 70:749-757.

Jauhari S, Singh S, Dash AK. Paclitaxel Profiles of Drug Substances, Excipients and Related Methodology,2009; 34:299-344

Jia L, Shen J, Zhang D, Duan C, Liu G, Zheng D, Tian X, Liu $\mathrm{Y}$, Zhang Q. In Vitro and in vivo evaluation of oridonin-loaded long circulating nanostructured lipid carriers. International journal of biological macromolecules, 2012; 50:523-529

Koning GA, Krijger GC. Targeted multifunctional lipid-based nanocarriers for image-guided drug delivery. Anti-cancer agents in medicinal chemistry, 2007; 7:425-440.

Kuo YC, Chung JF. Physicochemical properties of nevirapineloaded solid lipid nanoparticles and nanostructured lipid carriers. Colloids and surfaces B, Biointerfaces,2011; 83:299-306. 
Lala R, Awari N. Nanoemulsion-based gel formulations of COX-2 inhibitors for enhanced efficacy in inflammatory conditions. Applied Nanoscience, 2014; 4:143-151.

Liggins RT, Hunter W, Burt HM. Solid-state characterization of paclitaxel. Journal of pharmaceutical sciences, 1997; 86:1458-1463.

Liu D, Liu Z, Wang L, Zhang C, Zhang N. Nanostructured lipid carriers as novel carrier for parenteral delivery of docetaxel. Colloids and surfaces B, Biointerfaces, 2011; 85:262-269.

Liu J, Gong T, Wang C, Zhong Z, Zhang Z. Solid lipid nanoparticles loaded with insulin by sodium cholate-phosphatidylcholinebased mixed micelles: preparation and characterization. International journal of pharmaceutics, 2007; 340:153-162.

Lønning PE. Study of suboptimum treatment response: lessons from breast cancer. The lancet oncology, 2003 4:177-185.

Miao $\mathrm{J}$ et al., Improved cytotoxicity of paclitaxel loaded in nanosized lipid carriers by intracellular delivery. Journal of Nanoparticle Research,2015; 17:1-13.

Nair HB, Sung B, Yadav VR, Kannappan R, Chaturvedi MM, Aggarwal BB. Delivery of antiinflammatory nutraceuticals by nanoparticles for the prevention and treatment of cancer. Biochemical pharmacology, 2010; 80:1833-1843.

Narvekar M, Xue HY, Eoh JY, Wong HL. Nanocarrier for poorly water-soluble anticancer drugs--barriers of translation and solutions. AAPS PharmSciTech, 2014; 15:822-833.

Orive G, Anitua E, Pedraz JL, Emerich DF. Biomaterials for promoting brain protection, repair and regeneration. Nature Review of Neuroscience, 2009; 10:682-692.

Peer D, Margalit R. Fluoxetine and reversal of multidrug resistance. Cancer letters.2006; 237:180-187

Priya MK, Iyer PR. Anticancer studies of the synthesized gold nanoparticles against MCF-7 breast cancer cell lines. Applied Nanoscience.2015; 5:443-448.

Riehemann K, Schneider SW, Luger TA, Godin B, Ferrari M, Fuchs H. Nanomedicine challenge and perspectives. Angewandte Chemie, 2009; 48:872-897

Sanad RA, Abdelmalak NS, Elbayoomy TS, Badawi AA. Formulation of a novel oxybenzone-loaded nanostructured lipid carriers (NLCs). AAPS PharmSciTech, 2010; 11:1684-1694.

Swidan SA, Ghonaim HM, Samy AM, Ghorab MM. Comparative study of solid lipid nanoparticles and nanostructured lipid carriers for In Vitro Paclitaxel delivery. Journal of Chemical and Pharmaceutical Research, 2016; 8:482-493.

Sharma P, Ganta S, Denny WA, Garg S. Formulation and pharmacokinetics of lipid nanoparticles of a chemically sensitive nitrogen mustard derivative: Chlorambucil. International journal of pharmaceutics, 2009; 367:187-194.
Tiwari R, Pathak K. Nanostructured lipid carrier versus solid lipid nanoparticles of simvastatin: comparative analysis of characteristics, pharmacokinetics and tissue uptake. International journal of pharmaceutics, 2011; 415:232-243.

Velmurugan R, Selvamuthukumar S. Development and optimization of ifosfamide nanostructured lipid carriers for oral delivery using response surface methodology. Applied Nanoscience 2015; 1-15.

Viswanath DS, Ghosh T, Prasad DH, Dutt NV, Rani KY. Viscosity of liquids: theory, estimation, experiment, and data. Springer Science \& Business Media. 2007.

Xin D, Wang Y, Xiang. The use of amino acid linkers in the conjugation of paclitaxel with hyaluronic acid as drug delivery system: synthesis, self-assembled property, drug release, and In Vitro efficiency. Pharmaceutical research, 2010; 27:380-389.

Yajima T, Itai S, Takeuchi H, Kawashima Y. Determination of optimum processing temperature for transformation of glyceryl monostearate. Chemical and pharmaceutical bulletin, 2002; 50:1430-1433.

Yang XY, Li YX, Li M, Zhang L, Feng LX, Zhang N. Hyaluronic acid-coated nanostructured lipid carriers for targeting paclitaxel to cancer. Cancer Letters, 2013; 334:338-345.

Yao HJ, Ju RJ, Wang XX, Zhang Y, Li RJ, Yu Y, Zhang L, Lu WL. The antitumor efficacy of functional paclitaxel nanomicelles in treating resistant breast cancers by oral delivery. Biomaterials, 2011; 32:3285-3302.

Zeng N, Hu Q, Liu Z, Gao X, Hu R, Song Q, Gu G, Xia H, Yao L, Pang Z, Jiang X, Chen J, Fang L. Preparation and characterization of paclitaxel-loaded DSPE-PEG-liquid crystalline nanoparticles (LCNPs) for improved bioavailability. International journal of pharmaceutics, 2012; 424:58-66.

Zhang Y, Tang L, Sun L, Bao J, Song C, Huang L, Liu K, Tian Y, Tian G, Li Z, Sun H, Mei L. A novel paclitaxel-loaded poly $(\varepsilon-$ caprolactone)/poloxamer 188 blend nanoparticle overcoming multidrug resistance for cancer treatment. Acta biomaterialia, 2010; 6:2045-2052.

\section{How to cite this article:}

Swidan SA, Ghonaim HM, Samy AM, Ghorab MM. Efficacy and In Vitro Cytotoxicity of Nanostructured Lipid Carriers for Paclitaxel Delivery. J App Pharm Sci, 2016; 6 (09): 018-026. 